\title{
Kármán-Howarth closure equation on the basis of a universal eddy viscosity
}

\author{
F. Thiesset, ${ }^{1}$ R. A. Antonia, ${ }^{1}$ L. Danaila, ${ }^{2}$ and L. Djenidi ${ }^{1}$ \\ ${ }^{1}$ School of Engineering, University of Newcastle, NSW 2308 Callaghan, Australia \\ ${ }^{2}$ CORIA, UMR 6614, Avenue de l'Université, Boîte Postale 12, 76801 Saint Etienne du Rouvray, France
}

(Received 8 May 2013; published 29 July 2013)

\begin{abstract}
This Rapid Communication presents a simple closure for the two-point correlation transport equation in decaying isotropic turbulence. It relies essentially on an eddy viscosity $v_{t}$ which exhibits some remarkable universal facets over an impressively wide range of scales. This allows us to model the third-order structure functions in different decaying flows covering a large extent of Reynolds numbers. The model is numerically time integrated to predict the decay of second-order structure functions and compared to experiments in grid turbulence. Agreement between predictions and measurements is satisfactory.
\end{abstract}

DOI: 10.1103/PhysRevE.88.011003

PACS number(s): 47.27.em, 47.27.Jv

The Kármán-Howarth equation [1] can be written in terms of velocity structure functions [2]

$$
3 \partial_{t} \overline{(\Delta u)^{2}}=\frac{1}{r^{4}} \partial_{r}\left[r^{4}\left(6 v \partial_{r} \overline{(\Delta u)^{2}}-\overline{(\Delta u)^{3}}\right)\right]-4 \bar{\epsilon} .
$$

$\Delta u=u(x+r)-u(x)$ is the longitudinal velocity increment between two points separated by a distance $r$ and $\partial_{\alpha} \bullet=$ $\partial \bullet / \partial \alpha$. Further, $\bar{\epsilon}=15 v \overline{\left(\partial_{x} u\right)^{2}}$ is the mean dissipation rate with $v$ the kinematic viscosity, and the overbar denotes averaging. Second- and third-order structure functions $\overline{(\Delta u)^{2}}$ and $\overline{(\Delta u)^{3}}$ appearing in Eq. (1) are usually interpreted as the kinetic energy and the kinetic energy transfer at a given scale, respectively, two crucial quantities for modeling turbulent flows.

In spectral space, the equivalent equation known as Lin's equation [3] reads

$$
\partial_{t} E(k)=T(k)-2 \nu k^{2} E(k)
$$

in which $E(k)$ is the three-dimensional energy spectrum, $k$ is the wave number, and $T(k)$ is the spectral energy transfer function. Equation (2) describes essentially the same physical mechanism as Eq. (1), i.e., the decay, the transfer, and the dissipation of energy at a given scale or wave number.

In the last 50 years, several closures of Eq. (2) have been developed and are still extensively employed. Among others, we can cite the direct interaction approximation model proposed by Kraichnan [4] or the eddy damped quasinormal Markovian (EDQNM) closure [5].

On the contrary, closures of Eq. (1) have not received the same attention. To our knowledge, Millionshchikov [6] (in Russian), Domaradzki and Mellor [7], Effinger and Grossmann [8], Oberlack and Peters [9], and Baev and Chernykh ([10], and references therein) are the only authors who proposed a model (sometimes identical) for $\overline{(\Delta u)^{3}}$. All of them are based on the concept of an eddy viscosity $v_{t}$, i.e., Eq. (1) is then formally rewritten as

$$
3 \partial_{t} \overline{(\Delta u)^{2}}=\frac{1}{r^{4}} \partial_{r}\left[r^{4} 6\left(v+v_{t}\right) \partial_{r} \overline{(\Delta u)^{2}}\right]-4 \bar{\epsilon} .
$$

The third-order structure function is thus related to $v_{t}$ and $\overline{(\Delta u)^{2}}$ through

$$
\overline{(\Delta u)^{3}}=-6 v_{t} \partial_{r} \overline{(\Delta u)^{2}},
$$

where $v_{t}$ is a function of the separation $r$. Domaradzki and Mellor [7] proposed an expression for $v_{t}$ on the basis of inertial range asymptotic relations $\left(R_{\lambda} \rightarrow \infty\right.$, where $R_{\lambda}=\sqrt{\overline{u^{2}}} \lambda / \nu$ is the Reynolds number based on the Taylor microscale $\lambda \equiv$ $\sqrt{15 v \overline{u^{2}} / \bar{\epsilon}}$. However, as mentioned by the authors, the latter expression was not consistent with the scaling $\overline{(\Delta u)^{3}} \propto r^{3}$ as $r$ goes to zero. This constraint led Oberlack and Peters [9] to handle another expression for $v_{t}$, consistent with both dissipative and inertial range scaling laws. Here again, $v_{t}$ was parametrized through a constant (called $\kappa_{0}$ in their paper), the value of which relies on asymptotic inertial laws. Even though the use of asymptotic relations may be questionable in the context of finite Reynolds number flows (for instance, see [11], and references therein), both models were in satisfactory agreement with the third-order correlation functions measured by Stewart and Townsend [12] at (very) low Reynolds numbers $\left(R_{\lambda}<60\right)$.

This intriguing feature indicates that the assumption of infinite Reynolds numbers is not a necessary condition for asymptotic expressions of $v_{t}$ to be employed. Therefore, there is matter for investigating the approach towards the asymptote and the universal properties, i.e., the flow and $R_{\lambda}$ dependence, of the turbulent eddy viscosity, with the goal of providing an efficient simple closure scheme in physical space.

The results presented in this Rapid Communication highlight that the Kolmogorov normalized eddy viscosity reveals a remarkable degree of universality over a wide range of scales. An analytical expression for $v_{t}$ is provided revealing the existence of two universal parameters: the skewness of velocity derivative $S$ and a new scale of turbulence called $r_{c}$. In the inertial range and beyond, $v_{t}$ closely follows the asymptotic scaling even though neither $\overline{(\Delta u)^{2}}$ nor $\overline{(\Delta u)^{3}}$ indicate any unambiguous scaling. We then take advantage of these properties to model the third-order structure functions in different decaying flows, for Reynolds numbers $R_{\lambda}$ lying between 50 and 1100. Finally, the model is numerically time integrated to predict the decay of second-order structure functions and compared to experiments in grid turbulence $\left(R_{\lambda} \approx 50\right)$ for downstream distances up to $80 M$ ( $M$ is the grid mesh size).

In order to derive an analytical expression for $v_{t}$, we first recall that at small scales, $\overline{(\Delta u)^{2}}=\bar{\epsilon} r^{2} / 15 v$ and $\overline{(\Delta u)^{3}}=$ 
$-S\left(\bar{\epsilon} r^{2} / 15 v\right)^{3 / 2} . S=-\overline{\left(\partial_{x} u\right)^{3}} /\left[\overline{\left(\partial_{x} u\right)^{2}}\right]^{3 / 2}$ is the skewness of the longitudinal velocity derivative with respect to the longitudinal direction $x$. It follows that in the dissipative range

$$
\frac{v_{t}}{v}=\frac{S}{12 \sqrt{15}} r^{* 2} \text {. }
$$

Hereafter, the asterisk denotes normalization by the Kolmogorov scales, i.e., $r^{*}=r / \eta$ with $\eta=\left(v^{3} / \bar{\epsilon}\right)^{1 / 4}$. Second, in the context of infinite Reynolds numbers and for scales in the range $\eta \ll r \ll L$ ( $L$ is the integral length scale), $\overline{(\Delta u)^{2}}=C_{u}(\bar{\epsilon} r)^{2 / 3}$ and $\overline{(\Delta u)^{3}}=-A_{u} \bar{\epsilon} r\left(C_{u}=2, A_{u}=4 / 5\right.$ [13]). Hence, in the inertial range

$$
\frac{v_{t}}{v}=\frac{1}{5 C_{u}} r^{* 4 / 3}
$$

Equation (6) was already proposed by Domaradzki and Mellor [7], even though we became aware of this after we derived it. Following, e.g., [14], we match Eqs. (5) and (6) into a single expression

$$
\frac{v_{t}}{v}=\frac{S r^{* 2}}{12 \sqrt{15}\left[1+\gamma r^{* 2}\right]^{1 / 3}} .
$$

Equation (7) generalizes the expression of [7] by covering both dissipative and inertial ranges. In Eq. (7), the crossover length scale between dissipative and inertial range $r_{c}^{2}=$ $1 / \gamma$ is determined by equating Eqs. (5) and (6), yielding $r_{c}^{*}=\left(12 \sqrt{15} / 5 C_{u} S\right)^{3 / 2}$. As for the EDQNM spectral closure, dissipative and inertial range intermittency effects are not taken into account in the present analysis. According to the Kolmogorov theory [13], $S, C_{u}$, and consequently $r_{c}^{*}$ are universal. However, in the context of finite Reynolds number flows, $S$ and $r_{c}^{*}$ are (a priori) two free parameters. In the following, we turn our attention to their evolution with respect to the Reynolds number.

The analytical expression for $v_{t}$ [Eq. (7)] is thus compared to the one inferred from experiments in grid, wake, round, and plane jet turbulence. The Reynolds number is in the range $50 \leqslant$ $R_{\lambda} \leqslant 1100$. The grid turbulence experiments are described in [15]. The wake flow facility is described in [15], while experiments in the round and plane jets are outlined in [15]. For the wake, round, and plane jet experiments, the measurements were made at the centerline, thus avoiding to account for any additive production terms in Eq. (1) due to the mean shear.

The dependence on Reynolds number of the measured eddy viscosity is presented in Fig. 1(a). At small scales $\left(r^{*} \lesssim 10\right)$, all experimental points converge onto a single curve which is well represented by Eq. (5) with $S=0.424$ [Fig. 1(a)]. The value of $S$ used here is the mean value between the five experiments. $S$ varies by only $5 \%$ from one experiment to another. This indicates that the skewness of the velocity derivative $S$ remains constant in agreement with the Kolmogorov theory [13]. For the range of Reynolds numbers investigated, the effect of internal intermittency on $S$ [16] is not discernible. Both the constancy and the value itself of $S$ are quite consistent with all experimental values compiled by [17], at least for the same range of Reynolds numbers. Further, it is in perfect agreement with EDQNM [16].

As we progress through to the larger scales $\left(10 \lesssim r^{*} \lesssim\right.$ $10^{2}$ ), even though second- (not shown) and third-order

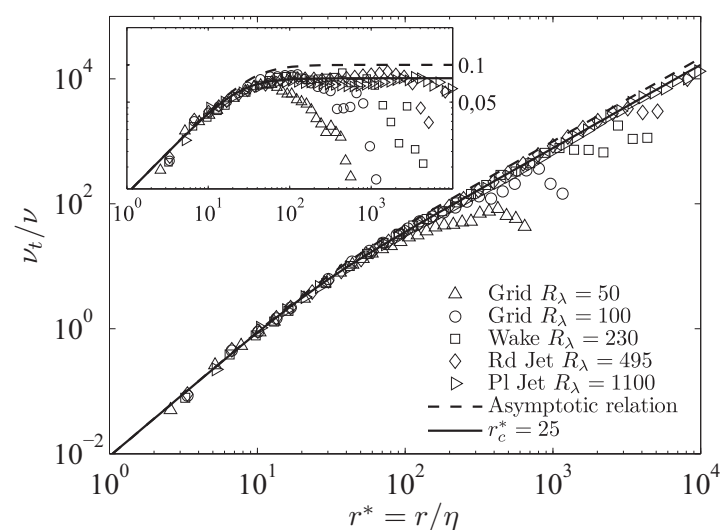

(a)

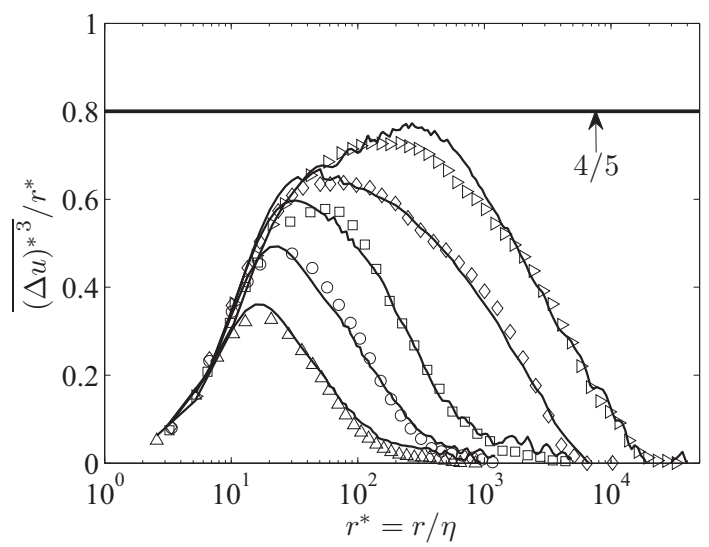

(b)

FIG. 1. (a) $v_{t} / v$ as a function of $r^{*}$ measured in different flows $\left(50 \leqslant R_{\lambda} \leqslant 1100\right)$. Equation (7) (dashed line); Eq. (7) with $r_{c}^{*}=$ 25 (solid line). The inset depicts the compensated eddy viscosity $\left(v_{t} / v\right) / r^{* 4 / 3}$. (b) Kolmogorov-normalized third-order structure functions. Symbols are the same as in Fig. 1(a); solid lines represent the present model using $r_{c}^{*}=25$.

structure functions [Fig. 1(b)] become $R_{\lambda}$ dependent, the eddy viscosity $v_{t}$ follows the same evolution independently of the Reynolds number. In other words, the Kolmogorov normalized eddy viscosity collapses over a wider range of separations in comparison to $\overline{\left(\Delta u^{*}\right)^{2}}$ and $\overline{\left(\Delta u^{*}\right)^{3}}$.

Then, for separations $r^{*} \gtrsim 10^{2}$, the effect of Reynolds number becomes discernible and the $r^{* 4 / 3}$ scaling range extends as the Reynolds number increases. Note that the separation beyond which the measured eddy viscosity differs from the prediction of Eq. (7) in Fig. 1(a) corresponds to the scale beyond which $\overline{(\Delta u)^{3 *}} / r^{*}$ is almost zero in Fig. 1(b). Therefore, $v_{t}$ remains universal in the range of separations over which the third-order structure function has to be modeled. We further observe that, though very close to the asymptotic relation, Eq. (7), a constant value of $r_{c}^{*}=25.0$ (instead of 36.3 providing $C_{u}=2$ ) is more suitable to parametrize $v_{t}$ over the whole range of Reynolds numbers. This supports a universal value for $r_{c}^{*}$, although weaker than the expected (Kolmogorov) value. This is in agreement with the observations of [7] revealing that the prefactor in Eq. (6) varies by only a few percent in the range $50 \leqslant R_{\lambda} \leqslant 10^{4}$ and remains always smaller than the expected asymptotic value even at a very high Reynolds number. 
Finally, the last observation that one can make is that at the highest Reynolds number $\left(R_{\lambda}=1100\right)$, the scaling $v_{t} \propto r^{* 4 / 3}$ is accurately satisfied over almost two decades of separations $\left(10^{2} \lesssim r^{*} \lesssim 10^{4}\right)$ while there is no unambiguous scaling range for either $\overline{(\Delta u)^{2}}$ (not shown) or $\overline{(\Delta u)^{3}}$ [Fig. 1(b)]. The scaling range of $v_{t}$ does not appear to be sensitive to any intermittency effect and is also much more extended than that of secondand third-order structure functions.

At this stage, we can draw the overall conclusion that, at least over the range of Reynolds numbers investigated here, $S$ and $r_{c}^{*}$ can be reasonably considered as universal. The constancy of $S$ relies on the validity of the Kolmogorov normalization in the dissipative range, which holds even at low Reynolds numbers [18]. In contrast, the constancy of $r_{c}^{*}$ is quite intriguing since it is now well known that the Kolmogorov "constant" $C_{u}$ and the scaling exponent of $\overline{(\Delta u)^{2}}$ are sensitive to the Reynolds number variations (at least for $R_{\lambda}<10^{4}$ [11]). To a large extent, the observed universality of $r_{c}^{*}$ is thus most likely due to some compensating effects that occur between $C_{u}$, $A_{u}$, and the scaling exponent of both $\overline{(\Delta u)^{2}}$ and $\overline{(\Delta u)^{3}}$ involved in Eq. (6). The consequence is that $r_{c}^{*}$ remains constant with respect to the Reynolds number.

The universality of $v_{t}$ can be further justified recalling that $v_{t}(r) \propto r^{2} / \tau(r)$ [see Eq. (19) in [19]], in which the characteristic time scale $\tau(r)$ is representative of the cascade mechanism. In spectral space, one possible expression for $\tau(k)$ is that of Batchelor and Kraichnan [20] that was recently invoked by [21] as a closure for the passive scalar spectral equation. In [20], $\tau(k)$ was interpreted as the time scale of the strain at a given wave number due to all larger scales. Using Kolmogorov scaling, $\tau(k)$ can be expressed as

$$
\tau^{*}\left(k^{*}\right) \propto\left[\int_{0}^{k^{*}} p^{* 2} E^{*}\left(p^{*}\right) d p^{*}\right]^{-1 / 2},
$$

where $p$ is a dummy integration variable. In Eq. (8), the normalized spectrum is multiplied by $p^{* 2}$ so that the contribution to the integral of the largest scales (low wave numbers) is weak. On the contrary, contributions from the smallest scales are magnified and the range of scales over which the Kolmogorov scaling is observed is extended [18]. In other words, the integrand $p^{* 2} E^{*}\left(p^{*}\right)$ in Eq. (8) always satisfies Kolmogorov scaling over a larger range of scales compared to $E^{*}\left(p^{*}\right)$ [18]. Therefore, since $v_{t}$ is intimately related to $\tau$ via $v_{t}(r) \propto r^{2} / \tau(r)$, the same conclusions can be drawn for the eddy viscosity.

The idea of invoking a set of scales which yields a collapse of velocity statistics over a wider range of scales was already used in [22] for which the relevant scales are $\lambda$ and $\overline{q^{2}}=\overline{u_{i} u_{i}}$ (twice the total kinetic energy). Further, in the energy-containing and inertial ranges, [23] demonstrated that the use of $\overline{u^{2}}$ and the von Kármán length scale $\left(\equiv \bar{u}^{2 / 2} / \bar{\epsilon}\right)$ leads to a satisfactory collapse of energy spectra. As far as the eddy viscosity is concerned, it appears that the relevant normalization is given by the Kolmogorov scales.

We now take advantage of this extended universality to develop a simple closure equation for Eq. (1). Thirdorder structure functions are thus calculated from measured second-order structure functions using Eqs. (4) and (7). The comparison between modeled and measured third-order structure functions is shown in Fig. 1(b).

Since Eq. (7) accurately represents the measured eddy viscosity, it is not surprising to observe that modeled and measured third-order structure functions are in excellent agreement [Fig. 1(b)]. The shape and evolution of $\overline{(\Delta u)^{3 *}} / r^{*}$ with respect to the Reynolds number are very well reproduced. The minor differences that may be observed are rather due to some slight errors in evaluating the derivative of measured second-order structure functions.

A much more stringent test of the validity of the present closure is the following. Starting with an initial condition at a particular position in the flow, can we reliably predict the decay of second-order structure functions downstream? To this end, Eq. (3) has to be time integrated.

Since theory is compared to a spatially decaying turbulence (in this case grid turbulence [15]), we relate the final time of integration to the downstream distance by means of Taylor's hypothesis, i.e., $x \equiv U t$ ( $U$ is the mean flow velocity). The time integration of Eq. (3) is handled using a fourth-order

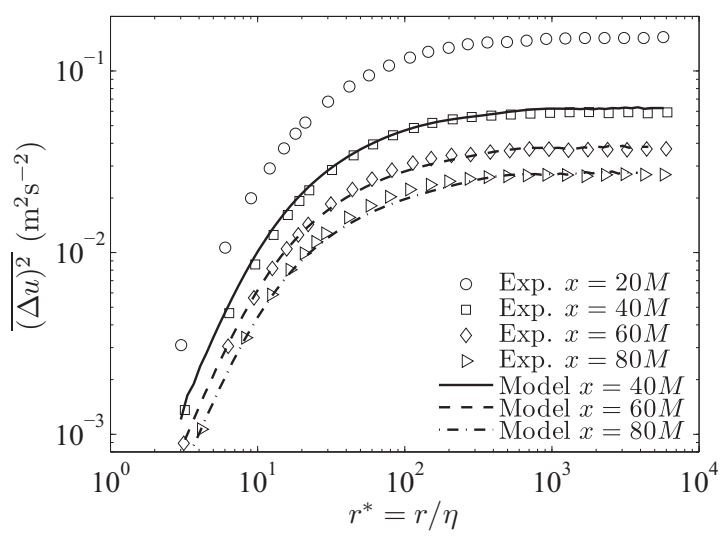

(a)

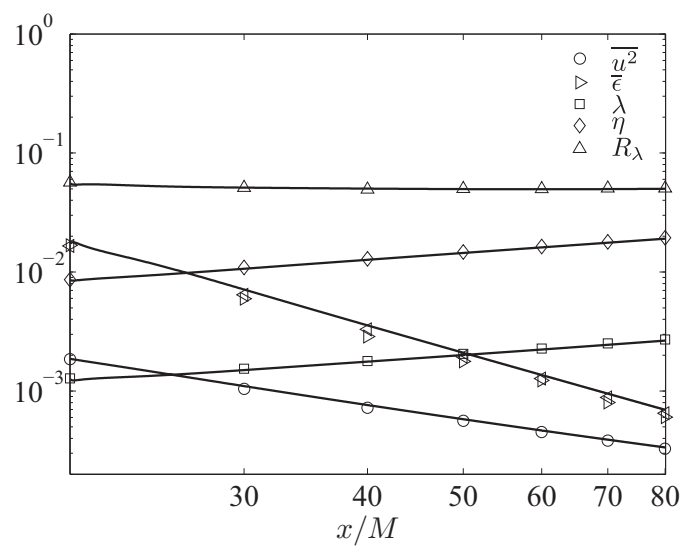

(b)

FIG. 2. (a) Comparison between measured and predicted secondorder structure functions in grid turbulence $\left(R_{\lambda} \approx 50\right)$. The time integration is started at $x=20 M$. (b) Evolution of $\overline{u^{2}} / U^{2}, \bar{\epsilon} M / U^{3}\left(10^{2}\right)$, $\lambda / M\left(10^{-2}\right), \eta / M$, and $R_{\lambda}\left(10^{-3}\right)$ with $x / M . U=6.4 \mathrm{~m} \mathrm{~s}^{-1}$ is the mean flow velocity. Symbols represent measured values, while solid lines are the predicted values. The mean dissipation rate $\bar{\epsilon}$ is estimated from the relation $\bar{\epsilon}=15 v \overline{\left(\partial_{x} u\right)^{2}}(\triangleright)$ and from Eq. (9) $(\triangleleft)$. The measured Taylor and Kolmogorov length scales were inferred from $\bar{\epsilon}$ computed from Eq. (9). 
Runge-Kutta algorithm. Derivatives $\partial_{r} \bullet$ are approximated by a central second-order finite difference scheme. Boundary conditions are set as $\overline{(\Delta u)^{2}}(r=0)=0$ and $\partial_{r} \overline{(\Delta u)^{2}}(r \rightarrow$ $\infty)=0$.

Results are given in Fig. 2(a). The initial conditions are set at $x=20 M$ behind the grid $(M=24.76 \mathrm{~mm}$ is the grid mesh size) and predictions are compared with measurements at $x=40,60$, and $80 M$. The initial Reynolds number $R_{\lambda}$ is about 50 and decreases slightly with $x$.

Measured and predicted second-order structure functions are in good agreement [Fig. 2(a)]. Minor differences can be observed at large separations where the model very slightly overestimates $\overline{(\Delta u)^{2}}$. From the decay of second-order structure functions, one can obtain the evolution of one-point statistics, i.e., the longitudinal velocity variance $2 \overline{u^{2}}=\overline{(\Delta u)^{2}}(r \rightarrow \infty)$, the mean dissipation rate $\bar{\epsilon}=15 v \lim _{r \rightarrow 0} \overline{(\Delta u)^{2}} / r^{2}$, the Taylor and Kolmogorov length scales ( $\lambda$ and $\eta$ ), and the Reynolds number $R_{\lambda}$. The mean dissipation rate can also be evaluated though the one-point energy budget

$$
\bar{\epsilon}=-\frac{1}{2} \partial_{t} \overline{q^{2}}
$$

where $\overline{q^{2}}=\overline{u^{2}}+\overline{v^{2}}+\overline{w^{2}}$ is twice the total kinetic energy. The evolution of one-point statistics is depicted in Fig. 2(b). The variation with respect to the downstream distance of all these quantities is globally very well reproduced by the present model. One can further note that the magnitude of the measured mean dissipation rate inferred from $\bar{\epsilon}=15 v \overline{\left(\partial_{x} u\right)^{2}}$ is smaller $(\approx 15 \%)$ than that predicted by the model. This discrepancy may be due to the smallest scales not being sufficiently resolved by the hot wire measurements. Indeed, values of $\bar{\epsilon}$ using Eq. (9) are only $\approx 10 \%$ smaller than those predicted.

The idea of predicting the decay of one-point statistics from a two-point closure equation was also tackled by Lohse [8], with a closure scheme based on the variable range mean field theory. In the latter study, the prediction of basic quantities, such as the normalized energy dissipation and enstrophy decay rates, compared favorably with experimental results in a particular type of decaying flow where the integral length scale does not vary with time. Obviously, this type of analytical treatment cannot be applied to decaying grid turbulence where the integral length scale grows continuously with time (or distance from the grid).

In summary, the universal facets of the eddy viscosity for the closure of the Kármán-Howarth equation are examined in detail. It is highlighted that $v_{t}$ remains impressively universal over a remarkable range of scales. An analytical expression for $v_{t}$ is further proposed, based on the observed constancy of the skewness of velocity derivatives and highlights the existence of a new scale of turbulence called $r_{c}$. The model is in good agreement with measurements in different types of decaying flows, over a wide range of Reynolds numbers. The closure scheme is finally time integrated and reproduces measured second-order structure functions in grid turbulence quite favorably.

The financial support of the "Agence Nationale de la Recherche" (ANR), under the project "ANISO," is gratefully acknowledged. R.A.A. and L.D. acknowledge the support of the Australian Research Council.
[1] T. von Kármán and L. Howarth, Proc. R. Soc. London, Ser. A 164, 192 (1938).

[2] G. K. Batchelor, Proc. Cambridge Philos. Soc. 43, 533 (1947); L. Danaila, F. Anselmet, T. Zhou, and R. A. Antonia, J. Fluid Mech. 391, 359 (1999).

[3] T. von Kármán and C. C. Lin, Adv. Appl. Mech. 2, 1 (1951).

[4] R. Kraichnan, J. Fluid Mech. 4, 497 (1959).

[5] S. Orszag, J. Fluid Mech. 41, 363 (1970).

[6] M. Millionshchikov, Pis'ma Zh. Exp. Teor. Fiz. 10, 406 (1969); 11, 203 (1970).

[7] J. A. Domaradzki and G. L. Mellor, J. Fluid Mech. 140, 45 (1984).

[8] H. Effinger and S. Grossmann, Z. Phys. B 66, 289 (1987); D. Lohse, Phys. Rev. Lett. 73, 3223 (1994).

[9] M. Oberlack and N. Peters, Applied Scientific Research 51, 533 (1993).

[10] M. K. Baev and G. G. Chernykh, J. Engineering Thermophysics 19, 154 (2010).

[11] R. A. Antonia and P. Burattini, J. Fluid Mech. 550, 175 (2006).

[12] R. W. Stewart and A. A. Townsend, Philos. Trans. R. Soc. London, Ser. A 243, 359 (1951).
[13] A. Kolmogorov, Dokl. Akad. Nauk. SSSR 30, 299 (1941); 125, 15 (1941).

[14] G. K. Batchelor, Proc. Cambridge Philos. Soc. 47, 359 (1951).

[15] T. Zhou, R. A. Antonia, L. Danaila, and F. Anselmet, Exp. Fluids 28, 143 (2000); R. A. Antonia, B. R. Pearson, and T. Zhou, Phys. Fluids 12, 3000 (2000); R. A. Antonia, M. Ould-Rouis, F. Anselmet, and Y. Zhu, J. Fluid Mech. 332, 395 (1997).

[16] W. J. T. Bos, L. Chevillard, J. Scott, and R. Rubinstein, Phys. Fluids 24, 015108 (2012).

[17] K. R. Sreenivasan and R. A. Antonia, Ann. Rev. Fluid Mech. 29, 435 (1997).

[18] R. A. Antonia, L. Djenidi, and L. Danaila (unpublished).

[19] L. Danaila, R. A. Antonia, and P. Burattini, Physica D 241, 224 (2012).

[20] G. K. Batchelor, J. Fluid Mech. 5, 113 (1959); R. H. Kraichnan, ibid. 47, 525 (1971).

[21] L. Danaila and R. A. Antonia, Phys. Fluids 21, 111702 (2009).

[22] W. George, Phys. Fluids 4, 1492 (1992).

[23] L. T. Adzhemyan, M. Hnatich, D. Horvath, and M. Stehlik, Phys. Rev. E 58, 4511 (1998). 